\title{
Minimum Bias and Underlying Event Studies at CDF
}

\author{
Niccolò Moggi ${ }^{* i \dagger}$ \\ INFN, Bologna \\ E-mail: moggi@bo.infn.it
}

Soft, non-perturbative, interactions are poorly understood from the theoretical point of view even though they form a large part of the hadronic cross section at the energies now available. We review the CDF studies on minimum-bias ad underlying event in $p \bar{p}$ collisions at $2 \mathrm{TeV}$. After proposing an operative definition of "underlying event", we present part of a systematic set of measurements carried out by the CDF Collaboration with the goal to provide data to test and improve the QCD models of hadron collisions. Different analysis strategies of the underlying event and possible event topologies are discussed. Part of the CDF minimum-bias results are also presented: in this sample, that represent the full inelastic cross-section, we can test simultaneously our knowledge of all the components that concur to form hadronic interactions. Comparisons with MonteCarlo simulations are always shown along with the data. These measurements will also contribute to more precise estimates of the soft QCD background of high- $p_{T}$ observables.

XVIII International Workshop on Deep-Inelastic Scattering and Related Subjects April 19 -23, 2010

Convitto della Calza, Firenze, Italy

\footnotetext{
*Speaker.

${ }^{\dagger}$ on behalf of the CDF Collaboration
} 


\section{Introduction and Definitions}

Soft hadron interactions are usually collected by a trigger called "Minimum-Bias" (MB) which actually selects events from all possible kind of interaction, proportionally to their production rate.

In order to avois ambiguities, we introduce an operational definition of "underlying event" (UE) as that part of the event final state that cannot be directly associated to the primary (perturbative) $2 \rightarrow 2$ parton-parton scattering. At least in hadronic collisions, with this definition the UE may be described as composed by the beam remnants, initial- and final-state gluon radiation and additional - with respect to the first hard one - parton-parton interactions (MPI).

Besides being an interesting research field in itself, soft interactions in general represent a background to high- $p_{T}$ rare processes in two ways: pile-up of MB collisions affects any measurement in high luminosity environments, while UE specifically affects some high- $p_{T}$ observables, such as jets, within a single interaction.

\section{The structure of Minimum-Bias and its Evolution with $Q^{2}$}

Because it represent the full cross section, the MB data sample offers the ground to study soft phenomena and their evolution toward hard interactions.

As was shown already in RunI [1], the MB sample has a jet structure which can be observed as an increase of the particle density around the high- $p_{T}$ ("leading") particle at $p_{T}$ as low as 0.5 $\mathrm{GeV} / \mathrm{c}$. The density of particles associated with the leading one (that defines a "toward" region in the event) is larger than the average density in MB [2]. Such jet structure is emphasized when rising the leading particle momentum. The evolution of the UE with the leading particle $p_{T}$ has been investigated for increasing momenta. To contrast the low statistics of higher momenta in MB, a jet sample was used at higher $Q^{2}$ ( $p_{T}$ of leading jet). These studies lead to a first tuning of the PYTHIA model on the CDF data ("tuneA") that includes a parameterization of MPI and that is still among the best in reproducing MB distributions, as we will show later.

\section{Underlying Event Measurements}

The $\eta-\phi$ regions orthogonal to the leading particle axis ("transverse") are very sensitive to the underlying event features since these regions contain only few of the particles originated by the hard parton scattering. Within the transverse region it is possible to define a MAX (MIN) region [3] as that with larger (smaller) particle activity. The $M A X$ corresponds to the region where most of gluon radiation is found. In the MIN the behavior of the beam-beam remnants (and MPI) is emphasized.

Our measurements, from a sample of $2.7 \mathrm{fb}^{-1}$, include charged particles in the range $p_{T}>0.5$ $\mathrm{GeV} / \mathrm{c}$ and $|\eta|<1$. Data are corrected to stable particles on the base of a MonteCarlo that includes full detector simulation. Particles are considered stable if $c \tau>10 \mathrm{~mm}$. Jets are selected with a MidPoint cone based algorithm with cone size of 0.7 and $f_{\text {merge }}=0.75$. For leading jet we require that it lies in the range $|\eta|<2$.

Cleaner measures may be obtained by selecting more definite event topologies. For example it is possible to suppress the $3^{\text {rd }}$ jet and require only two back-to-back jets to supress gluon radiation. 

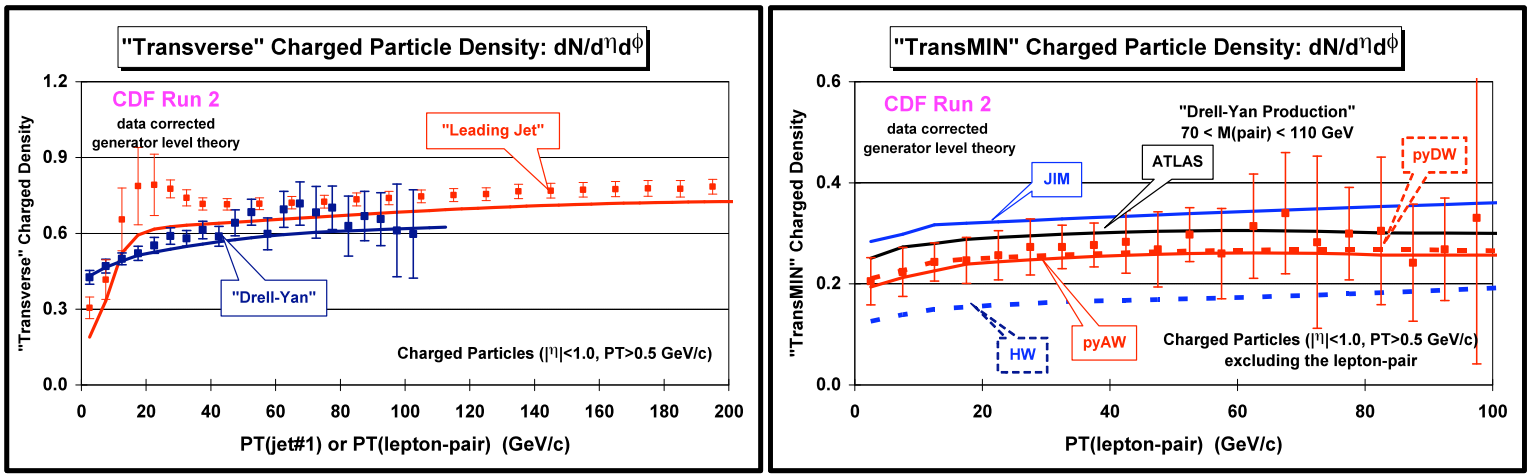

Figure 1: Left: charged particle density in the transvese region for leading-jet and Drell-Yan events. Right: charged particle density in the MIN region for Drell-Yan events.
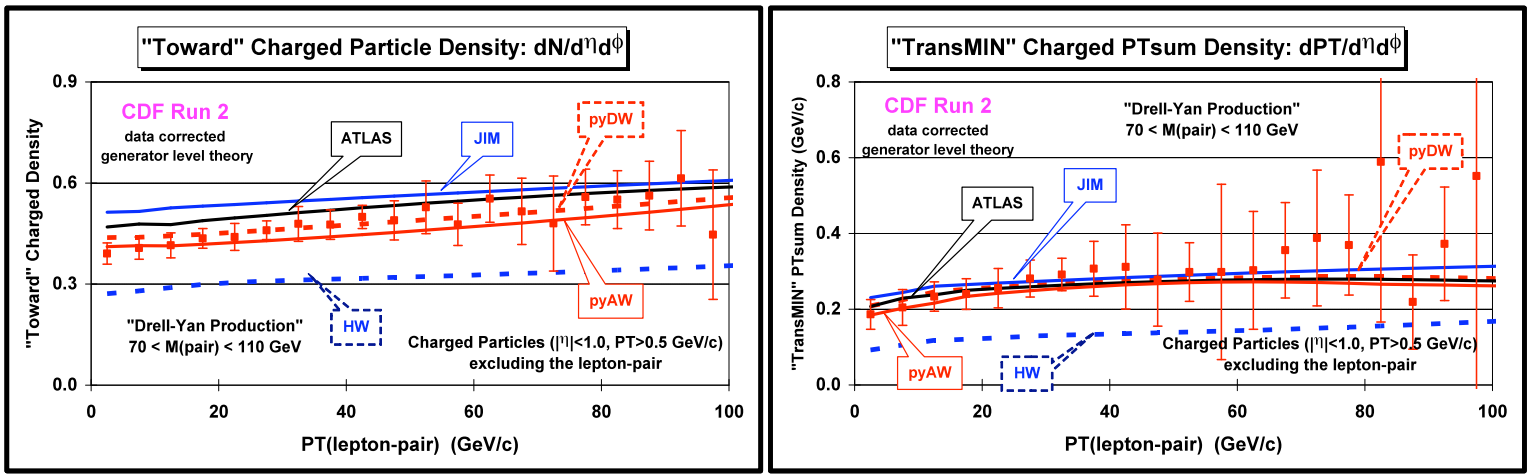

Figure 2: Left: charged particle density in the Toward region for Drell-Yan events. Right: the sum of charged particle $p_{T}$ in the $M I N$ region for Drell-Yan events.

More interestingly, we analyze events with a pair of "Drell-Yan" leptons of $p_{T}>20 \mathrm{GeV} / \mathrm{c}$ in the mass region $70<M_{\text {pair }}<110 \mathrm{GeV} / \mathrm{c}^{2}$ with $\left|\eta_{\text {pair }}\right|<6$ [4]. The final state particles - excluding the lepton pair - are studied as a function of the pair invariant mass and the lepton pair direction replaces the leading jet direction. Unlike for high- $p_{T}$ jets, here there is no final-state gluon radiation, therefore both the transverse and toward regions are very sensitive to the UE. Data shows that particle activity still increase with the pair $p_{T}$ in the $M A X$ region while the $M I N$ region is flat. Observables specifically sensitive to radiation may be found in a region defined as the difference in contents of $M A X-M I N$, for any chosen observable.

The CDF Collaboration has carried out a systematic set of measurements of various observables in different event topologies and compared them to different MC generators [5] and/or tunings (see table 3). For many of all the possible combinations, the various regions defined above have been analized with the goal to provide data to be used to test and improve the QCD models that are being used in hadron collisions. Only few examples can be shown here. Fig.1 (left) shows the charged particle density $d N / d \eta d \phi$ in the transverse region $(M A X+M I N)$ in leading-jet and DrellYan events (DY). The MIN region is shown on the right for DY only. The particle $\sum p_{T}$ density $\sum p_{T} / d \eta d \phi$ is shown on Fig.2 right. Fig.2 left shows the toward region in DY events.

We may already draw some conclusions. The UE is similar in DY and leading-jet events; 
Pyhtia tuneA (tuneAW) does a good job in describing the UE observables, although the agreement is not always perfect; in general, all models with MPI agree better with data than models without.

\begin{tabular}{||l|c||}
\hline $\begin{array}{l}\text { observables } \\
\text { topologies } \\
\text { models }\end{array}$ & $d N / d \eta d \phi, d p_{T} / d \eta d \phi, \sum p_{T} / d \eta d \phi,\left\langle p_{T}\right\rangle, p_{T}^{M A X}$ \\
leading-jet, back-to-back jet, Drell-Yan \\
PYTHIA tuneA, tune AW, tuneDW; HERWIG, HERWIG+JIMMY etc... \\
\hline
\end{tabular}

Table 1: Incomplete summary of the observables, the event topologies and the MonteCarlo configurations that have been studied.

\section{Minimum-Bias Measurements}

The MB final state observables that are experimentally accessible represent a complicated mix of different physics effects ranging from purely soft to very hard ones. The description of MB requires therefore, besides the 'ordinary' QCD parton scattering, also the knowledge of the correct mixture of soft and hard processes and a reliable description of the other components of the interaction. For this reason MB is the sample where to perform a global test of our models.

At CDF the MB trigger is implemented by means of two sets of Cherenkov counters placed on both sides of the detector and requires a coincidence of both signals. The result is that the MB sample contains most of the inelastic cross section with a small contamination of single- and double-diffractive. The data sample consists of about $506 \mathrm{pb}^{-1}$ with low instantaneous luminosity in order to avoid large pile-up of events. This sample was corrected for the trigger efficiency and the contamination of diffractive events was subtracted so that we deal with inelastic data.

The experimental selection for charged particles is similar as in the UE studies. However, the lower $p_{T}$ acceptance was set to $0.4 \mathrm{GeV} / \mathrm{c}$ to compare with RunI measurements. Data were fully corrected to particle level using a PYTHIA tuneA MC sample. Effects of the residual pile-up of collisions were also corrected for.

The CDF Collaboration has realized a large set of measurements in the MB sample. Here we show the charged particle multiplicity $\left(N_{c h}\right)$ distribution and the event $\sum E_{T}$ spectrum (Fig.3). The $N_{c h}$ distribution represent the probability of observing an interaction with $N_{c h}$ (charged) primary particles in the final state. The data presented here have the highest precision and the largest range extension ever reached in the pseudorapidity range $|\eta|<1$. This makes of the new measurement an important handle for the extrapolation of MC models to LHC energies. For example in [6] small variations in PYTHIA tunes at $2 \mathrm{TeV}$ produce large variations in the high multiplicity tail of the distributions at $14 \mathrm{TeV}$.

The measurement of the event transverse energy sum [7] represents a first attempt at describing the full final state including neutral particles. In this regard, it is complementary to the charged particle measurement in describing the global features of the inelastic $p \bar{p}$ cross section. The differential cross section $d^{3} \sigma /\left(\Delta \phi \Delta \eta d E_{T}\right)$ for $|\eta|<1$ is shown in Fig. 3 (right).

In neither case the PYTHIA simulation closely reproduce the data over the whole spectrum. In the $\sum E_{T}$ the peak of the MC distribution is slightly shifted to higher energies with respect to the data. Systematic uncertainties are large and do not allow more definite statements. 

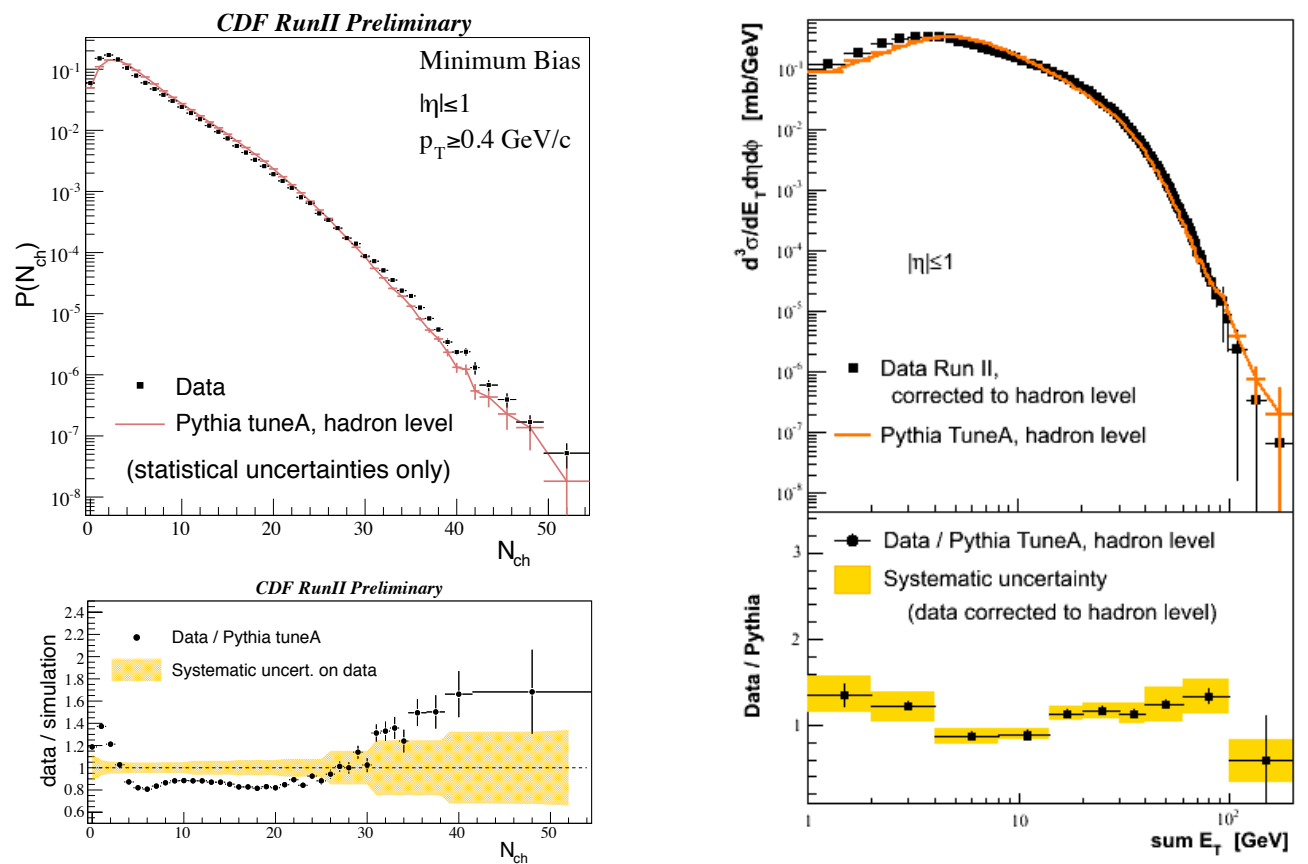

Figure 3: The $N_{c h}$ and $\sum E_{T}$ distributions in MB compared to PYTHIA tuneA simulation. Data is corrected to hadron level.

\section{Conclusions}

The CDF Collaboration has performed a systematic set of measrements of UE and MB. The data presented here can be used to improve QCD Monte Carlo models and further our understanding of the mechanisms that concur to the formation of hadronic final states and of their interplay.

We have shown that the UE is very similar in different event topologies and that a mechanism of multiple parton interactions is very useful in order to reproduce high multiplicity final states with the correct transverse momenta. PYTHIA tuneA (and similar configurations) reproduces the data at $2 \mathrm{TeV}$, at least qualitatively.

The increased precision measurements of the MB sample are an important handle for the extrapolation of MC models to LHC energies and will contribute to more precise estimates of soft QCD background in high- $p_{T}$ measurements.

\section{References}

[1] T.Affolder et al. (CDF Collaboration), Phys.Rev. D65, 092002 (2002).

[2] R.Field, Min-Bias At The Tevatron, AIP Conf.Proc.928:91-98 (2007).

[3] R.Field, Physics at TeV colliders 275-426, Les Houches 2001, hep-ph/0204316

[4] T.Aaltonen et al. (CDF Collaboration), submitted to Phys.Rev. D, arXiv:1003.3146.

[5] R.Field, Physics at the Tevatron, FERMILAB-CONF-06-530-E (2006).

[6] P.Z.Skands, The Perugia Tunes, arXiv:1005.3457.

[7] Niccolò Moggi, "Study of Soft QCD at the Tevatron", "Nuovo Cimento" 32C, N 5-6, (2010) 113. 